\title{
Improved Efficiency of Thin Film a-Si:H Solar Cells with
}

\section{$\mathrm{Au}$ Nanoparticles}

\author{
Kazi Islam ${ }^{1}$, Aaesha Alnuaimi ${ }^{1}$, Ali Kemal Okyay ${ }^{2}$ and Ammar Nayfeh ${ }^{1}$ \\ ${ }^{1}$ Institute Center for Future Energy Systems (iFES), Masdar Institute of Science and Technology, \\ PO BOX 54224, Abu Dhabi, UAE \\ ${ }^{2}$ UNAM, Bilkent University, Ankara, Turkey
}

\begin{abstract}
In this work, the effect of Au nanoparticles on the performance of a-Si:H solar cells is investigated experimentally. Au nanoparticles of 10, 20, 50, 80, 100, 200 and $400 \mathrm{~nm}$ are spin coated on ITO before metallization. The results show an increase in the $\mathbf{J}_{\mathrm{sc}}$ and efficiency with increasing nanoparticle size. The $\mathbf{J}_{\mathrm{sc}}$ increases from $9.34 \mathrm{~mA} / \mathrm{cm}^{2}$ to $10.1 \mathrm{~mA} / \mathrm{cm}^{2}$. In addition, the efficiency increases from $4.28 \%$ to $5.01 \%$.
\end{abstract}

Index Terms —nanoparticles, photovoltaics, plasmonics, thin film, solar cells, amorphous $\mathrm{Si}$

\section{INTRODUCTION AND MOTIVATION}

Effective light trapping mechanisms are important for the improved performance of thin film solar cells. For enhanced absorption in thin film solar cells, different approaches such as surface texturing and back reflectors have been described in [1-2]. More recently, nanotechnology has been used with plasmonic light trapping of metal nanoparticles (NP) like gold $\mathrm{Au})$ or silver $(\mathrm{Ag})$ [3-5]. For incorporating metal NPs into solar cells, different methods have been established that includes island annealing and colloidal metal particles [6-7]. Also, some numerical models have been developed to understand the plasmonic effect [8]. In this work, the effect of $\mathrm{Au}$ nanoparticles on the performance of thin film a-Si:H n-i-p solar cells is studied.

\section{PLASMONIC ENHANCEMENT}

Surface plasmons are collective oscillations of the free charges at a metal boundary. Metals support surface plasmons, either localized as for metal nanoparticles or propagating in case of planar metal surfaces. By controlling the size and shape of the metallic nanostructures, the surface plasmon resonance or plasmon propagating properties can be varied. Since the surface resonances of metals are mostly in the visible or in the infrared region of the electromagnetic spectrum, they are of particular interest for photovoltaic application. Two prominent mechanisms can explain the contribution of metallic nanoparticles based on application: scattering mechanism and the near-field localization effect [9].

Photocurrent enhancement by metal nanoparticles on the top surface of solar cells can be explained by the light scattering mechanism. Metal nanoparticles are strong scatterers of light at wavelengths near their resonant frequency [10].

A point dipole model can describe the absorption and scattering of the incoming light by the nanoparticls for particles with diameters well below the wavelength the incoing light. The scattering and absorption cross-sections are given by the following equations [10]:

$$
\begin{gathered}
C_{\text {scat }}=\frac{1}{6 \pi}\left(\frac{2 \pi}{\lambda}\right)^{4}|\alpha|^{2} \\
C_{a b s}=\frac{2 \pi}{\lambda} \operatorname{Im}[\alpha]
\end{gathered}
$$

where

$$
\alpha=3 V\left[\frac{\left[{ }^{\varepsilon_{p}} / \varepsilon_{m}-1\right.}{{ }^{{ }^{p}} / \varepsilon_{m}+2}\right]
$$

is the polarizability of the particle. Here $V$ is the particle volume, $\varepsilon_{p}$ is the dielectric function of the particle and $\varepsilon_{m}$ is the dielectric function of the embedding medium. From (3), its quite obvious that at $\varepsilon_{p}=-2 \varepsilon_{m}$ the particle polarizability will become very large. This very concept is termed as the surface plasmon resonance.
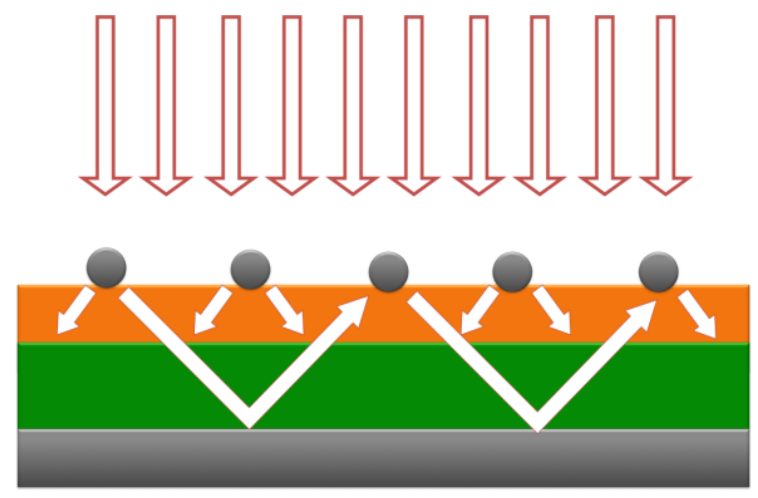

Fig. 1. Scattering of incoming light due to nanoparticles on top of ITO. (Figure not to scale)

At the surface plasmon resonance, the scattering crosssection exceeds the geometrical cross section of the nanoparticles e.g. silver nanoparticles in air has a scattering cross-section that is approximately ten times the crosssectional area of the particle at the resonant frequency[11]. In such a case, a substrate covered with a $10 \%$ areal density of 
nanoparticles can fully absorb and scatter the incoming light, to first-order [11][12]. For light trapping it is important that scattering is more efficient than absorption, a condition that is met for comparatively larger nanoparticles as can be seen from (1) and (2) [4].

Nanoparticle size, shape and distribution over the surface will determine the effect of the nanoparticles on the performance of the photovoltaic device [13].

\section{StRUCTURE AND FABRICATION PROCESS}

Fig. 2 shows the structure of the fabricated a-Si:H n-i-p solar cell. The structure is a stack of $80 \mathrm{~nm} \mathrm{ITO,} 20 \mathrm{~nm}$ heavily doped n-type a-Si, $500 \mathrm{~nm}$ undoped a-Si and finally $20 \mathrm{~nm}$ heavily doped $\mathrm{p}$-type a-Si on a p+-type $\mathrm{Si}$ wafer. The p+ Si substrate serves as the back contact, and does not contribute significantly to the carrier generation. After the ITO etch, Au NP of various sizes in solution were spin-coated.

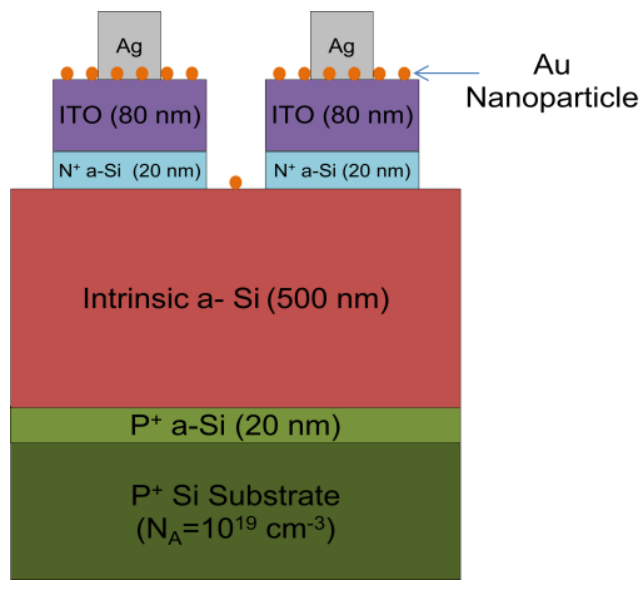

Fig. 2. Cross-section of the fabricated ITO/a-Si:H solar cell with $\mathrm{Au}$ nanoparticles deposited on the top surface. (Figure not to scale)

Seven different diameter-size, $10 \mathrm{~nm}, 20 \mathrm{~nm}, 50 \mathrm{~nm}, 80 \mathrm{~nm}$, $100 \mathrm{~nm}, 200 \mathrm{~nm}$ and $400 \mathrm{~nm}$ particle sizes were investigated. The spin coating recipe was optimized to obtain uniform distribution and high concentration of particles on the surface, simultaneously and was set to $2000 \mathrm{rpm}, 1000$ acceleration and spinning for 60 seconds. Fig. 4 is an SEM image of the top surface showing the Au NP on the ITO.

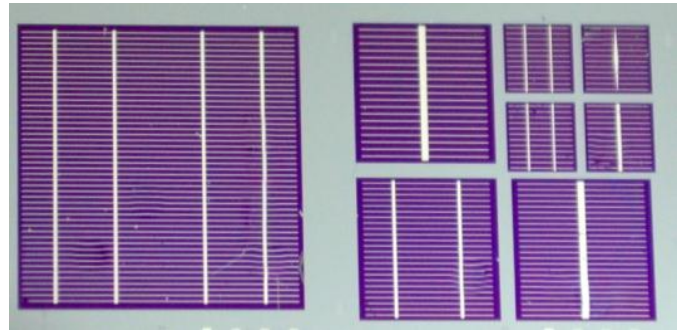

Fig. 3. Top view photograph of the fabricated solar cell.
After the NP coating, $100 \mathrm{~nm}$ of silver was deposited using Thermal Physical Vapor Deposition (PVD) tools. Three different cell area-sizes were fabricated i.e. $1 \mathrm{~cm} \times 1 \mathrm{~cm}, 0.5$ $\mathrm{cm} \times 0.5 \mathrm{~cm}$ and $0.25 \mathrm{~cm} \times 0.25 \mathrm{~cm}$. Fig. 3 shows the top view of final solar cell structure fabricated. Unless otherwise stated, solar cells of size $0.25 \mathrm{~cm} \times 0.25 \mathrm{~cm}$ are presented in this work.

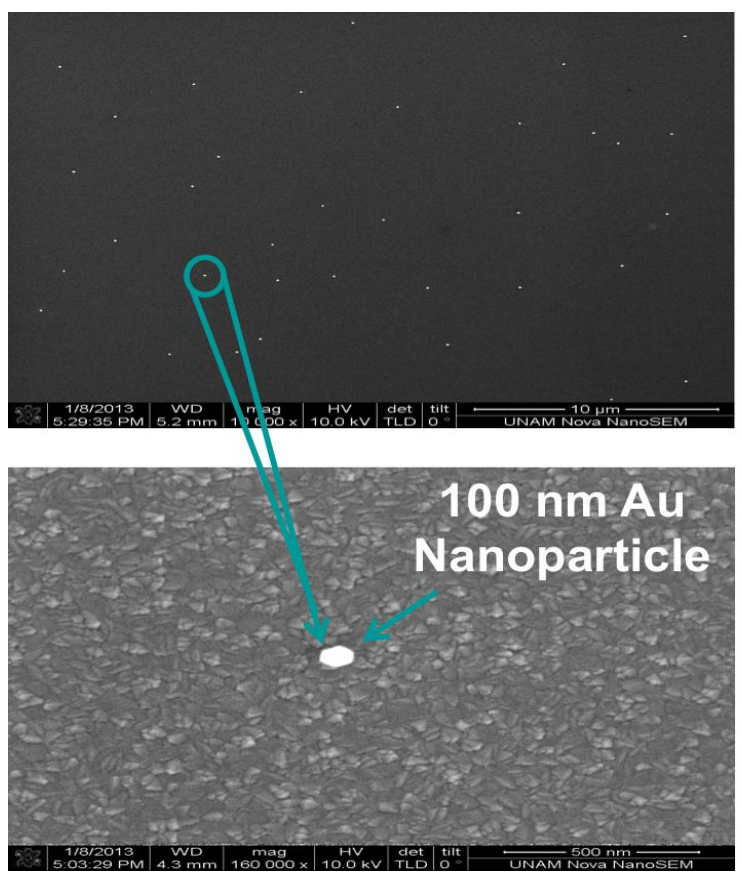

Fig. 4. SEM image of the top surface of the fabricated n-i-p a-Si:H solar cell with $100 \mathrm{~nm}$ Au nanoparticles on the ITO surface.

\section{EXPERIMENTAL RESULTS AND ANALYSIS}

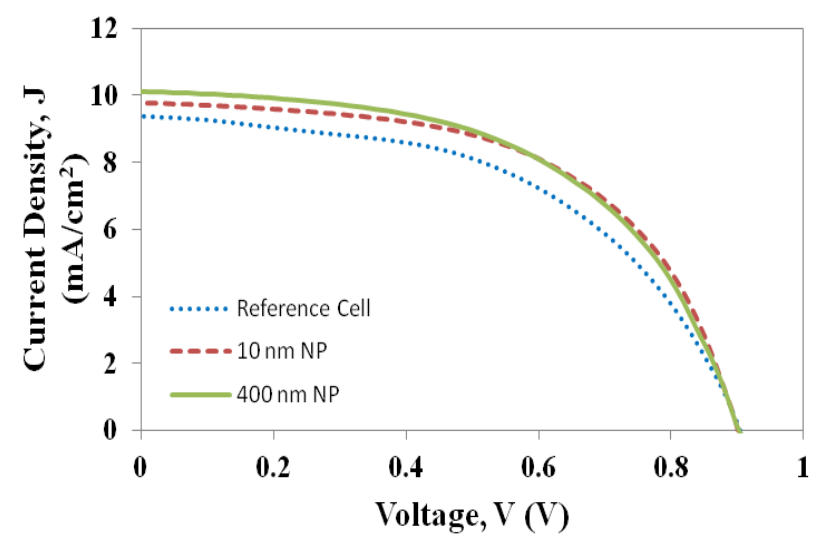

Fig. 5. Measured J-V characteristics of n-i-p a-Si:H reference cell without nanoparticles and cells with $10 \mathrm{~nm}$ and $400 \mathrm{~nm} \mathrm{Au}$ nanoparticles. 
Fig. 5 shows the J-V curve for the reference cell without NP and cells with $10 \mathrm{~nm}$ and $400 \mathrm{~nm} \mathrm{NP}$. The $\mathrm{V}_{\mathrm{oc}}$ is constant at $0.89 \mathrm{~V}$ while the $\mathrm{J}_{\mathrm{sc}}$ improves with larger NP size. Fig. 6 plots $\mathrm{J}_{\mathrm{sc}}$ vs. NP size. The $\mathrm{J}_{\mathrm{sc}}$ increases from $9.34 \mathrm{~mA} / \mathrm{cm}^{2}$ to 10.1 $\mathrm{mA} / \mathrm{cm}^{2}$. Fig. 7 plots the median reflectance across all wavelengths vs. NP size. The reflectance drops from $23.19 \%$ with no Au NP to less than $20 \%$ for all NP sizes. The drop shows the improved surface scattering due to the $\mathrm{Au}$ nanoparticles.

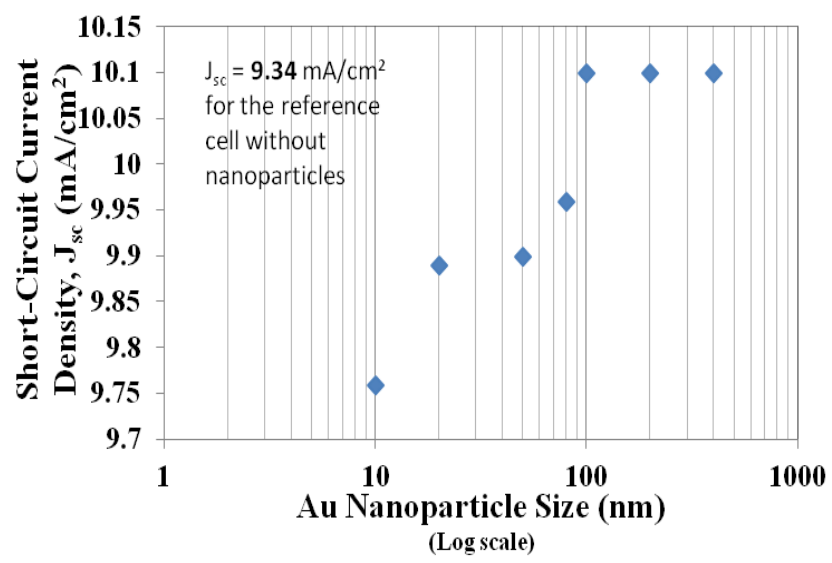

Fig. 6. $\mathrm{J}_{\mathrm{sc}}$ vs. Au nanoparticle size (plotted in log scale) for $\mathrm{Au}$ nanoparticle enhanced plasmonic n-i-p a-Si:H solar cells.

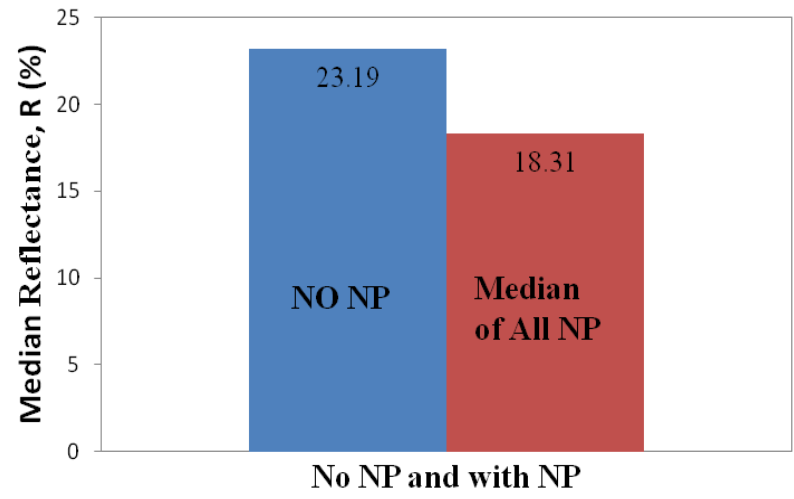

Fig. 7. Median reflectance vs. Au nanoparticle size (plotted in $\log$ scale) for $\mathrm{Au}$ nanoparticle enhanced plasmonic n-i-p a-Si:H solar cells.

Fig. 8 plots the efficiency vs. NP size. The efficiency increases with increasing NP size due the increase in the $\mathrm{J}_{\mathrm{sc}}$. The efficiency increases from $4.28 \%$ to $5.1 \%$. The $\mathrm{V}_{\text {oc }}$ does not change with NP size. The fill factor (FF) increases from $51 \%$ for the reference cell up to $56 \%$ for the Au NP based cell. For the most part the FF does not change with NP size.

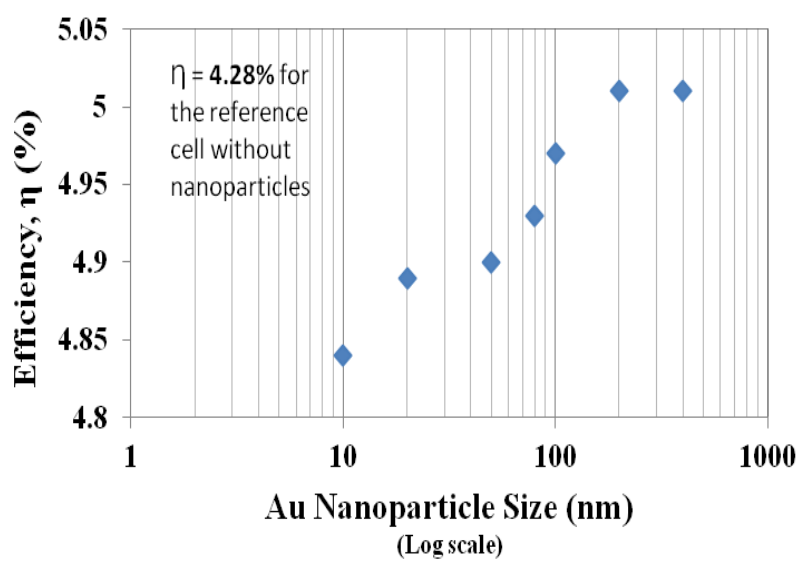

Fig. 8. Efficiency vs. Au nanoparticle size (plotted in log scale) for Au nanoparticle enhanced plasmonic n-i-p a-Si:H solar cells.

Fig. 9 plots the External Quantum Efficiency (EQE) of the reference cell, $10 \mathrm{~nm}$ and $400 \mathrm{~nm}$ Au NPs. The results show an improved spectral response with increasing NP size. The peak $\mathrm{EQE}$ is $59 \%$ for the cell with $400 \mathrm{~nm} \mathrm{NP}$ while the reference cell has $43 \%$ peak EQE. The EQE predicts that there should be a $41.6 \%$ increase in the $\mathrm{J}_{\mathrm{sc}}$ for the $400 \mathrm{~nm} \mathrm{NP}$ cell with respect to the no NP cell. From the actual measurement, the $\mathrm{J}_{\mathrm{sc}}$ increases from $9.34 \mathrm{~mA} / \mathrm{cm}^{2}$ for the no NP cell to 10.13 $\mathrm{mA} / \mathrm{cm}^{2}$ for the $400 \mathrm{~nm} \mathrm{NP}$ cell, which is $8.46 \%$ increment. More investigations are required to further explain this mismatch.

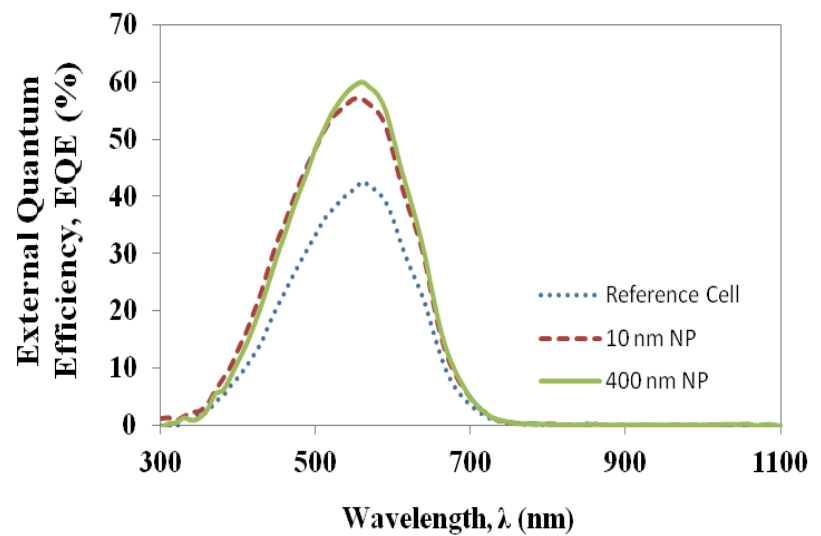

Fig. 9. Measured external quantum efficiency of n-i-p a-Si:H reference cell without nanoparticles and cells with $10 \mathrm{~nm}$ and 400 $\mathrm{nm}$ Au nanoparticles.

Table I summarizes the main findings of this work, listing the $\mathrm{J}_{\mathrm{sc}}$ and efficiency of the solar cells with respect to the $\mathrm{Au}$ NP size. The $\mathrm{J}_{\mathrm{sc}}$ increases by $8.46 \%$ with the introduction of NP for the best case and the efficiency improves by $17.06 \%$ with NP for the best case as well. Both the $\mathrm{J}_{\mathrm{sc}}$ and efficiency tend to improve with larger NP sizes. 
TABLE I

SUMMARY OF RESULTS

\begin{tabular}{|c|c|c|}
\hline Au NP Size $(\mathrm{nm})$ & $J_{\mathrm{sc}}\left(\mathrm{mA} / \mathrm{cm}^{2}\right)$ & $\eta(\%)$ \\
\hline 0 & 9.34 & 4.28 \\
\hline 10 & 9.76 & 4.84 \\
\hline 20 & 9.89 & 4.89 \\
\hline 50 & 9.9 & 4.9 \\
\hline 80 & 9.96 & 4.93 \\
\hline 100 & 10.1 & 4.97 \\
\hline 200 & 10.11 & 5.01 \\
\hline 400 & 10.13 & 5.01 \\
\hline
\end{tabular}

\section{CONCLUSIONS}

In summary, the effect of $\mathrm{Au}$ nanoparticles on the performance of a-Si:H thin film solar cells was studied. The results show an increase in $\mathrm{J}_{\mathrm{sc}}$, efficiency and spectral response with the $\mathrm{Au}$ nanoparticles. The results highlight a promising and simple enhancement for future thin film solar cells using nanoparticles.

\section{ACKNOWLEDGEMENT}

We would like to thank the students and the technical staff of UNAM at Bilkent University, Turkey for their help with the fabrication. We gratefully acknowledge financial support for this work provided by the Masdar Institute of Science and Technology.

\section{REFERENCES}

[1] M. Berginski et al. "Recent development on surfacetextured $\mathrm{ZnO}$ : $\mathrm{Al}$ films prepared by sputtering for thin-film solar cell application." Thin Solid Films 516.17 (2008): 5836-5841.

[2] V. E. Ferry et al. "Improved red-response in thin film a-Si: $\mathrm{H}$ solar cells with soft-imprinted plasmonic back reflectors." Applied physics letters 95.18 (2009): 183503183503.

[3] H. A Atwater and Albert Polman. "Plasmonics for improved photovoltaic devices." Nature materials 9.3 (2010): 205-213.

[4] K. R. Catchpole, and Albert Polman. "Plasmonic solar cells." Optics express 16.26 (2008): 21793-21800.

[5] Y. A Akimov, K. Ostrikov, and E. P. Li. "Surface plasmon enhancement of optical absorption in thin-film silicon solar cells." Plasmonics 4.2 (2009): 107-113.

[6] F.J. Beck, A. Polman, and K. R. Catchpole. "Tunable light trapping for solar cells using localized surface plasmons." Journal of Applied Physics 105.11 (2009): 114310-114310.

[7] D. Derkacs, S. H. Lim, P. Matheu, W. Ma, E.T. Yu, "Improved performance of amorphous silicon solar cells via scattering from surface plasmon polaritons in nearby metallic nanoparticles," Applied Physics Letters, 89(9), 093103-093103 (2006)

[8] Yu. A Akimov, W. S. Koh, and K. Ostrikov. "Enhancement of optical absorption in thin-film solar cells through the excitation of higher-order nanoparticle plasmon modes." Optics express 17.12 (2009): 10195-10205.

[9] Temple, T. L., G. D. K. Mahanama, H. S. Reehal, and D. M. Bagnall. "Influence of localized surface plasmon excitation in silver nanoparticles on the performance of silicon solar cells." Solar Energy Materials and Solar Cells 93, no. 11 (2009): 1978-1985.

[10] Bohren, Craig F., and Donald R. Huffman. Absorption and scattering of light by small particles. Wiley-Vch, 2008.

[11] B.J.Soller and D.G Hall, "Scattering enhancement from an array of interacting dipoles near a planar waveguide," JOSA B, 19(10), 2437-2448 (2002)

[12] K.R Catchpole and S. Pillai. "Absorption enhancement due to scattering by dipoles into silicon waveguides." Journal of applied physics 100.4 (2006): 044504-044504.

[13] Kelly, K. Lance, et al. "The optical properties of metal nanoparticles: the influence of size, shape, and dielectric environment." The Journal of Physical Chemistry B 107.3 (2003): 668-677. 